\title{
A importância das redes de suporte social no controle do processo saúde-doença
}

\section{The importance of social support networks in controlling the health-disease process}

DOI: $10.46919 / \operatorname{archv1n6-011}$

Recebimento dos originais: 01/09/2020

Aceitação para publicação: 30/10/2020

\section{Mariana Aquino Holanda Pinto}

Médica graduada pela Universidade de Fortaleza; especialização em Saúde da Família pela Universidade Federal do Ceará (Curso de especialização, pesquisa e inovação em Saúde da Família - CEFISP 2019). Endereço: Av. Washington Soares, 1321 - Edson Queiroz, Fortaleza - CE, 60811-905

E-mail: marianaholandap@gmail.com

\section{Laerte Canedo Ornelas Filho}

Médico graduado pela Universidade Federal do Ceará. Endereço: R. Cinco, 100 - Pres. Kennedy, Fortaleza - CE, 60355-636

E-mail:laerte.ornelas@gmail.com

\section{João Gabriel Oliveira Sousa}

Acadêmico de medicina: Centro Universitário Christus

Endereço: R. João Adolfo Gurgel, 133 - Cocó, Fortaleza - CE, 60190-180

E-mail: joaogabriel_oss7@ hotmail.com

\begin{abstract}
RESUMO
As redes de suporte social são sistemas que oferecem informação, apoio financeiro e emocional às pessoas em suas diferentes necessidades, atuando como um possível instrumento terapêutico e promotor da saúde. Esse trabalho tem como objetivo identificar, em um seio familiar, os problemas de saúde, os fatores de risco determinantes do processo saúde/doença e a eficácia dos apoios disponíveis na promoção da saúde. A pesquisa foi feita por meio de entrevistas semanais e da aplicação dos instrumentos Genograma, APGAR familiar e Ecomapa com a finalidade de conhecer a história dessa família, representar graficamente a estrutura e o padrão de repetição das relações familiares, medir a satisfação dos membros da família em relação aos elementos fundamentais para a unidade familiar e identificar os apoios disponíveis e sua influência no processo saúde-doença. Foi avaliada uma família composta por oito membros. O pai possuía histórico de etilismo e de tabagismo desde os 12 anos, tendo convívio familiar pouco funcional. $\mathrm{O}$ pai começou a frequentar a igreja, tendo melhorando seu relacionamento com a família e abandonado seus vícios e iniciou trabalho na instituição UNIFOR, conseguindo alguns benefícios. A Igreja possibilitou a modificação dos hábitos do Pai, que afetavam a relação familiar e a instituição Unifor, com o auxílio-saúde, permitiu-lhe maior informação sobre sua doença e melhor adesão ao plano terapêutico. Observou-se a influência das redes de suporte social no aumento da qualidade de vida e na promoção da saúde dessa família, interferindo no controle do processo saúde doença.
\end{abstract}

Palavras-chave: Redes de Suporte Social, Promoção da Saúde, Família.

\begin{abstract}
Social support networks are systems that offer information, financial and emotional support to people in their different needs, acting as a possible therapeutic tool and health promoter. This work aims to identify, within a family, health problems, risk factors determining the health / disease process and the
\end{abstract}


effectiveness of the support available in health promotion. The research was carried out through weekly interviews and the application of the Genogram, APGAR family and Ecomapa instruments with the purpose of knowing the history of this family, graphically representing the structure and repetition pattern of family relationships, measuring the satisfaction of family members in relation to the fundamental elements for the family unit and to identify the support available and its influence on the health-disease process. A family composed of eight members was evaluated. The father had a history of alcoholism and smoking since he was 12 years old, having a poorly functioning family life. The father started attending church, having improved his relationship with the family and abandoned his addictions and started working at the UNIFOR institution, obtaining some benefits. The Church made it possible to modify the Father's habits, which affected the family relationship and the Unifor institution, with health assistance, allowed him more information about his illness and better adherence to the therapeutic plan. It was observed the influence of social support networks in increasing the quality of life and promoting the health of this family, interfering in the control of the health-disease process.

Keywords: Social Support Networks, Health promotion, Family.

\section{INTRODUÇÃO}

Nas últimas décadas, o capitalismo globalizado tem acentuado as desigualdades sociais em diversos aspectos e contribuído para os agravos de saúde da população, que vem buscando, cada vez mais, além dos atendimentos médicos tradicionais, redes de suporte social, como grupos religiosos, trabalhos filantrópicos em organizações não governamentais, movimentos sociais e associações comunitárias dentre outros para sanar seus problemas em saúde e amenizar o sofrimento (CASTEL, 1993; BOURDIEU, 1998; VALLA, 1999; 2001).

As redes de suporte social são aliadas quando se trata de promoção à saúde e se configuram atualmente como formas terapêuticas fundamentais para atenção e cuidado integral à saúde (KOINONIA, 2009).

Em comunidades carentes, as instituições religiosas frequentemente se configuram como o primeiro local de busca por apoio pela população diante das dificuldades encontradas. Muitos de seus membros recorrem inicialmente aos seus líderes quando possuem alguma enfermidade, por exemplo, para que os auxiliem no direcionamento a um atendimento especializado, configurando essas lideranças como pontes entre o doente e a unidade de saúde (KOINONIA, 2009).

As instituições de ensino superior com formação na área da saúde e que disponibilizam de atendimentos clínicos para os pacientes funcionam como moderadores em saúde ao facilitar o acesso da população a serviços de saúde especializados sem custos (ALVARENGA et al, 2011), sanando as necessidades dos usuários, reduzindo as esperas e facilitando o fluxo no Sistema Único de Saúde (SUS).

Observando esses fatos, esse estudo foi realizado em um seio familiar, com o objetivo de identificar os problemas de saúde, os fatores de risco determinantes do processo saúde/doença e a eficácia dos apoios disponíveis na promoção da saúde. 
Para isso, foram utilizados os instrumentos: Genograma, APGAR familiar e Ecomapa.

O Genograma, tem sido utilizado como instrumento em Terapia Familiar Sistêmica (TFS) como forma eficiente de obter informações da constituição familiar. São registrados dados de importância para o indivíduo, tais como separações, doenças, mortes, acidentes, cirurgias e internações (MUNIZ e EISENSTEIN, 2009). Assim, são retratos gráficos da história e do padrão familiar, que identificam a estrutura básica, o funcionamento e os relacionamentos da família, evidenciando estressores e constituindo um mapa relacional do paciente e sua família.

O APGAR da família propõe a avaliação da funcionalidade familiar independentemente da fase do ciclo de vida dos seus membros (SILVA et al, 2014). Esse instrumento possibilita a detecção de disfunções no sistema familiar, viabilizando a fundamentação de intervenções voltadas a restabelecer o equilíbrio das relações existentes nesse sistema. Seu acrônimo APGAR provém da língua Inglesa e avalia a satisfação de cada membro quanto à Adaptação (Adaptation), Participação (Partneship), Crescimento (Growth), Afeição (Affection) e Resolução (Resolve) no seio familiar (ROCHA et al, 2002) .

O Ecomapa é um instrumento de abordagem familiar que possui o objetivo de avaliar a anatomia da família assim com o Genograma, sendo um diagrama das relações entre a família e a comunidade. Desenha o sistema ecológico onde a família e/ou o indivíduo estão incluídos, identificando seus padrões de organização e a natureza des suas relações com o meio onde habita e expondo o balanço entre seus recursos e necessidades (NASCIMENTO et al, 2014).

Essa pesquisa tem como base os princípios éticos da Declaração de Helsinki, redigida pela Associação Médica Mundial em 1964, que tem como objetivo fornecer orientações aos médicos e outros participantes em pesquisas clínicas envolvendo seres humanos (DINIZ e CORREA, 2001).

\section{DESENVOLVIMENTO}

Este trabalho avaliou uma família moradora da comunidade do Dendê na cidade de Fortaleza CE, por meio de entrevistas semanais no mês de novembro de 2012, utilizando-se do Genograma (Figura 1), do APGAR (Tabela 1) e do Ecomapa (Figura 2) como instrumentos normativos para diagnóstico adequado das relações interpessoais e das relações com o meio. O núcleo familiar era composta por oito membros. Seus integrantes eram o pai, paciente índice, de 55 anos de idade, etilista e tabagista desde os 12 anos de idade e portador de Diabetes Mellitus tipo II, diagnosticado aos 45 anos de idade e com má adesão terapêutica; mãe de 45 anos de idade; filho de 29 anos de idade; nora de 26 anos de idade; 2 netas de 8 e 3 anos de idade e 2 filhas de 24 e 21 anos de idade. 
Figura 1. Genograma do núcleo familiar.

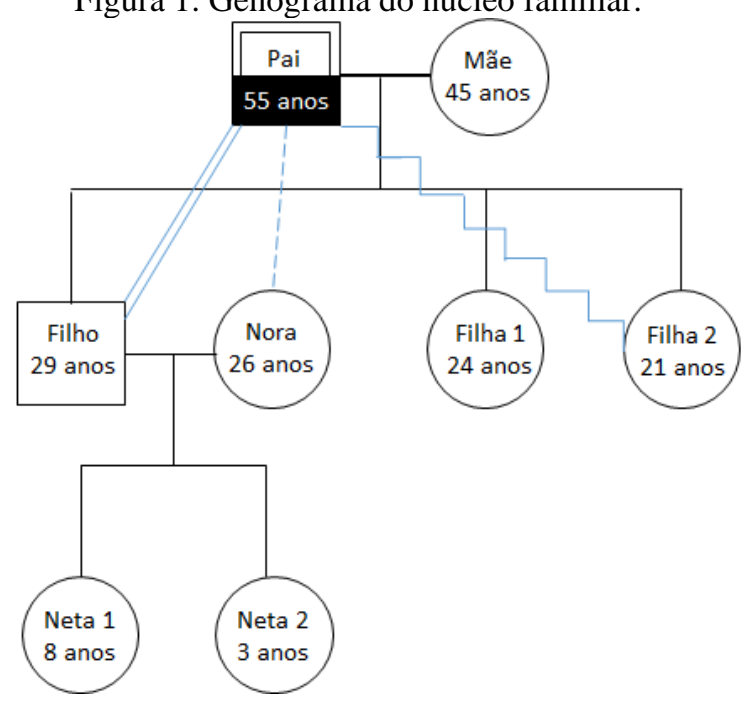

O pai, servidor da Universidade de Fortaleza - UNIFOR, tinha acompanhamento médico garantido pelo plano de saúde fornecido pela empresa e utilizava dos serviços e da estrutura que essa instituição fornecia aos funcionários e aos moradores da região onde se insere, no bairro Edson Queiroz. Apesar do acesso facilitado aos serviços especializados em saúde, tinha seu tratamento não efetivo por má adesão terapêutica decorrente do etilismo e do tabagismo e considerava seu convívio familiar com moderada disfunção, somando 5 pontos pelo APGAR (Tabela 1). Houve um ponto importante de ruptura do padrão de convívio social e familiar a partir do ano de 2006; neste período, influenciado pelo filho, o pai passou a frequentar uma comunidade evangélica no mesmo bairro onde mora e, a partir desta inserção no grupo social, obteve sucesso na modificação de seus hábitos, identificando que o abandono do tabagismo e do etilismo nesse período fora essencial para melhoria da relação familiar, ficando classificada como altamente funcional, alcançando 8 pontos na reaplicação do APGAR.

Tabela 1. APGAR familiar com os resultados do $1^{\circ}$ e do $2^{\circ}$ questionários.

\begin{tabular}{|c|c|c|c|}
\hline \multicolumn{2}{|c|}{} & $1^{\circ}$ Questionário & $2^{\circ}$ Questionário \\
\hline $\begin{array}{c}\text { Estou satisfeito(a) com a } \\
\text { atenção que recebo da } \\
\text { minha família quando } \\
\text { algo está me } \\
\text { incomodando. }\end{array}$ & $\begin{array}{c}\text { Quase sempre } \\
\text { Às vezes } \\
\text { Raramente }\end{array}$ & 1 & 2 \\
\hline $\begin{array}{c}\text { Estou satisfeito(a) com a } \\
\text { forma que minha família } \\
\text { discute as questões de } \\
\text { interesse comum e } \\
\text { compartilha comigo a } \\
\text { resolução dos problemas. }\end{array}$ & $\begin{array}{c}\text { Auase sempre } \\
\text { Raramente }\end{array}$ & 0 & 1 \\
\hline
\end{tabular}




\begin{tabular}{|c|c|c|c|}
\hline $\begin{array}{c}\text { Sinto que minha família } \\
\text { aceita meus desejos de } \\
\text { iniciar novas atividades } \\
\text { ou de realizar mudanças } \\
\text { em meu estilo de vida. }\end{array}$ & $\begin{array}{c}\text { Quase sempre } \\
\text { Às vezes } \\
\text { Raramente }\end{array}$ & 2 & 2 \\
\hline $\begin{array}{c}\text { Estou satisfeito(a) com a } \\
\text { maneira com que minha } \\
\text { família expressa afeição } \\
\text { e reage em relação aos } \\
\text { meus sentimentos de } \\
\text { raiva, tristeza e amor. }\end{array}$ & $\begin{array}{c}\text { Quase sempre } \\
\text { Às vezes } \\
\text { Raramente }\end{array}$ & 1 & 1 \\
\hline $\begin{array}{c}\text { Estou satisfeito(a) com a } \\
\text { maneira com que eu e } \\
\text { minha família passamos } \\
\text { o tempo juntos. }\end{array}$ & $\begin{array}{c}\text { Quase sempre } \\
\text { Às vezes } \\
\text { Raramente }\end{array}$ & 1 & 2 \\
\hline Total & & 05 pontos & 08 pontos \\
\hline
\end{tabular}

A comunidade do Dendê, pertencente ao bairro Edson Queiroz, na cidade de Fortaleza segue perfil de desenvolvimento e ocupação urbana registrados, a partir da década de 90, com especulação imobiliária, dispersão das classes média e alta para região sudeste com criação de aparatos muito voltados para estas classes e pouco preparados para atender a população de baixa renda, formando-se assim, como em toda a cidade de Fortaleza, um compartilhamento do espaço geográfico sem qualquer partilha dos instrumentos sociais entre as classes (MATOS e CARLEAL, 2003).

As oportunidades surgidas com a construção de shoppings, mercados e faculdades que atendessem a região gerou, ao mesmo tempo, oportunidades de trabalho na região e crescimento desorganizado com precarização da moradia, aumento de índices de criminalidade e saturação dos aparatos e serviços públicos. A comunidade viu então crescer a influência dos equipamentos de saúde e cidadania disponibilizados pela Universidade de Fortaleza - UNIFOR (MOURA et al. 2010), bem como das organizações religiosas que exercem, dentro da comunidade, papel civilizatório para além da espiritualidade, com redes apoio ao usuário de entorpecentes ou álcool e redes mediadoras de conflitos. O relacionamento entre a família e as redes de suporte social de seu bairro, ilustrado no ECOMAPA (Figura 2) interferiu de maneira relevante no aumento da qualidade de vida e na promoção de saúde dessa família, intervindo no controle do processo saúde-doença. 
Figura 2. ECOMAPA familiar.

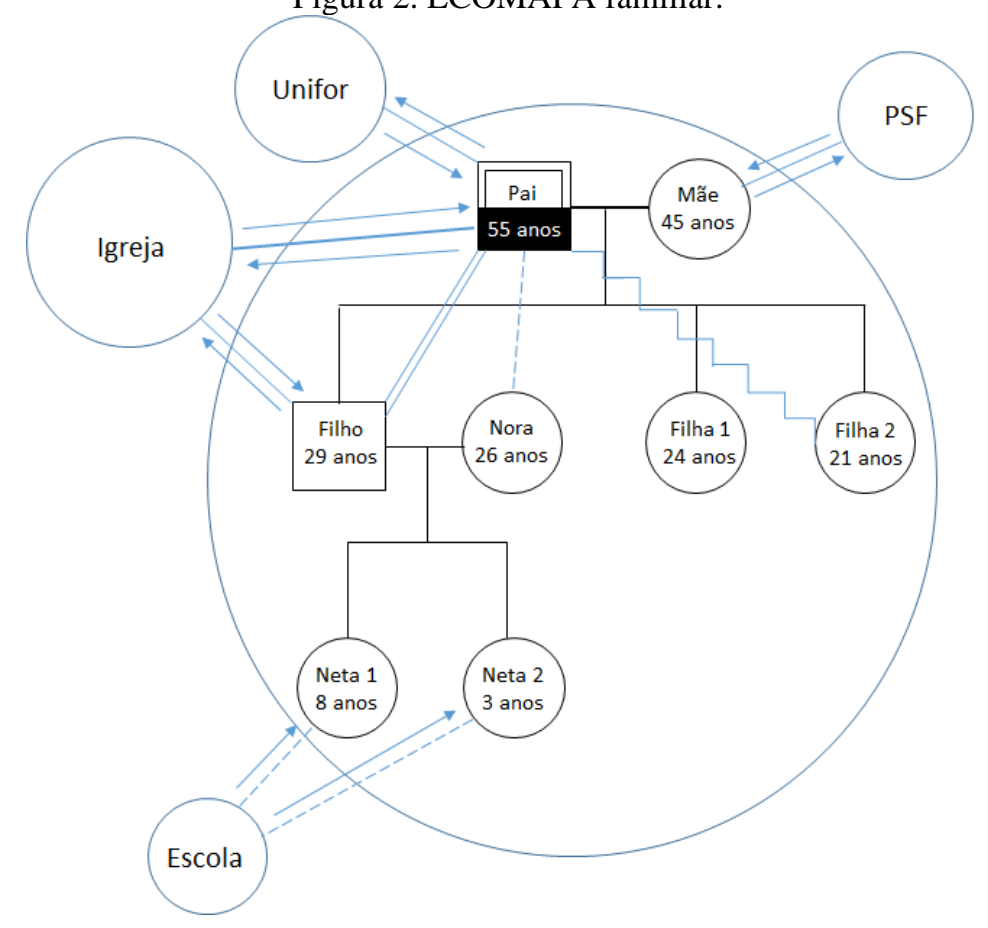

\section{CONCLUSÃO}

A participação das redes de apoio social pôde ser verificada a partir do ponto de inflexão gerado pelo contato do patriarca com comunidades religiosas locais e pelo suporte social e de saúde proporcionado por entidades privadas. Esta influência tem se tornado cada vez mais importante em comunidades fragilizadas e em famílias em estado de vulnerabilidade, quando o aparato estatal não se faz suficiente ou universal como se propõe.

A partir deste projeto notamos de forma objetiva a interdependência entre o processo de saúdedoença e o estado de bem estar sócio-cultural. Esta percepção ampara-se ainda na concepção de saúde como para além da presença ou ausência de patologia orgânica, mas como por um completo bem estar social, cultural, físico e espiritual.

\section{REFERÊNCIAS}

1. ALVARENGA, Márcia Regina Martins et al . Rede de suporte social do idoso atendido por equipes de Saúde da Família. Ciênc. saúde coletiva, Rio de Janeiro, v. 16, n. 5, p. 2603-2611, May 2011. Available from \&lt;http://www.scielo.br/scielo.php?script=sci_arttext\&amp;pid=S141381232011000500030\&amp;lng=en\&amp;nrm=iso\&gt; $\quad$ access $\quad$ on $12 \quad$ Oct. 2020. https://doi.org/10.1590/S1413-81232011000500030. Bairros de Fortaleza. Scripta Nova. [Rev Electrónic Barcelona]. 2003;7(146). Disponível em: <http://www.

2. BOURDIEU, P. Contrafogos: Táticas para Enfrentar a Invasão Neoliberal. Rio de Janeiro: Jorge Zahar, 1998. 
3. CASTEL, R. Da indigência à exclusão, a desfiliação: precariedade do trabalho e vulnerabilidade relacional. In: LANCETTI, A. (Org.). SaúdeLoucura 4. São Paulo: Hucitec, 1993 p. 21-48.

4. DINIZ, Debora; CORREA, Marilena. Declaração de Helsinki: relativismo e vulnerabilidade. Cad.Saúde Pública, Rio de Janeiro , v. 17, n. 3, p. 679-688, June 2001 . Available from \&lt;http://www.scielo.br/scielo.php?script=sci_arttext\&amp;pid=S0102-

311X2001000300022\&amp;lng=en\&amp;nrm=iso\&gt;. access on 12 Oct. 2020. Geografía cienc sociales. Barcelona: Universidad de http://dx.doi.org/10.1590/S0102-311X2001000300022.

5. KOINONIA, [s. 1.]. As redes de apoio social constituídas por pastores e agentes comunitários de saúde evangélicos no âmbito do cuidado e atenção à saúde da população. v. 16, ed. 4, 2009. DOI 1981-1810. http://www.koinonia.org.br/tpdigital/detalhes.asp?cod_artigo=318\&amp;cod_boletim=17\&amp;tipo= Artigos. Acesso em: 11 out. 2020.

6. MATOS AM, CARLEAL AN. Opulência e Miséria nos

7. MOURA, Karol Silva de; BESSA, Olívia Andréa Alencar Costa; NUTO, Sharmênia de Araújo Soares; SÁ, Henrique Luis do Carmo; VERAS, Fátima Maria Fernandes; BRAGA, José Ueleres. Projeto Coorte Dendê: diagnóstico demográfico e condições de moradia de uma comunidade de baixa renda em fortaleza, ceará. Revista Brasileira em Promoção da Saúde, [S.L.], v. 23, n. 1, p. 18-24, 2010. Fundacao Edson Queiroz. http://dx.doi.org/10.5020/18061230.2010.p18. Disponível em: https://periodicos.unifor.br/RBPS/article/view/1166/2288. Acesso em: 14 out. 2020.

8. MUNIZ, José Roberto; EISENSTEIN, Evelyn. Genograma: informações sobre família na (in)formação médica. Rev. bras. educ. med., Rio de Janeiro , v. 33, n. 1, p. 72-79, Mar. 2009. Available from\&lt;http://www.scielo.br/scielo.php?script=sci_arttext\&amp;pid=S010055022009000100010\&amp;lng=en\&amp;nrm=iso\&gt; $\quad$ access on 12 Oct. 2020.https://doi.org/10.1590/S0100-55022009000100010.

9. NASCIMENTO, Lucila Castanheira et al. GENOGRAMA E ECOMAPA:CONTRIBUIÇÕES DA ENFERMAGEM BRASILEIRA. Texto Contexto Enferm,Florianópolis, 23(1), p. 211-20, 2014.

10. ROCHA, Semiramis Melani Melo; NASCIMENTO, Lucila Castanheira; LIMA, Regina Aparecida Garcia de. Enfermagem pediátrica e abordagem da família: subsídios para o ensino de graduação. Rev. Latino-Am. Enfermagem, Ribeirão Preto, v. 10, n. 5, p. 709-714, Oct. 2002 . Available from $<$ http://www.scielo.br/scielo.php?script=sci_arttext\&pid=S010411692002000500013\&lng=en\&nrm=iso>. access on 11 Nov. 2020. https://doi.org/10.1590/S010411692002000500013.

11. SILVA, Maria Josefina da et al . Análise das propriedades psicométricas do APGAR de família com idosos do nordeste brasileiro. Esc. Anna Nery, Rio de Janeiro , v. 18, n. 3, p. 527-532, Sept. 2014 . Available from \&lt;http://www.scielo.br/scielo.php?script=sci_arttext\&amp;pid=S141481452014000300527\&amp;lng=en\&amp;nrm=iso\&gt; $\quad$ access $\quad$ on 12 Oct. 2020. http://dx.doi.org/10.5935/1414-8145.20140075. ub.es/geocrit/sn/sn-146(030).htm>

12. VALLA, V. V. (org.). O que a saúde tem a ver com a religião. Religião e cultura popular. Rio de Janeiro: DP\&A, 2001, pp. 113-139.

13. VALlA, V. V. Educação popular, saúde comunitária e apoio social numa conjuntura de globalização. Cadernos de Saúde Pública,v., 15, 1999, pp.7-14. 